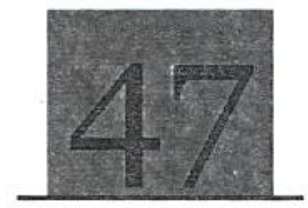

\title{
Tilapia sex reversal using methyl testosterone (MT) and its effect on fish, man and environment
}

\author{
Megbowon, I. / Mojekwu, T. O.
}

\section{Summary}

Tilapia is the second leading aquatic crop globally, next to carps and its production has increased tremendously within the last decade. Its production is however affected by stunting, a phenomenon brought about by overpopulation due to precocious reproduction under mixed sex culture system. Several methods such as use of predatory fish, hybridization, polyploidy and sex reversal, using methyl testosterone have been employed with different degrees of success. This paper reviews the use of methyl testosterone in sex reversal of tilapia and its consequent effects on fish, man and environment. It established the preference of all-male tilapia due to better and more uniform growth under culture system. It has been demonstrated that the hormone does not have any adverse effect on fish flesh after cessation of treatment of tilapia fry. In like manner, ingestion of fish produced by sex reversal does not therefore harm man. However, one is not too sure of the effect of the hormone or it's by-products on vital organs (liver, kidney, pancreas and gills), metabolic profile and nucleic acids. This may also affect muscle building and perceived libido-enhancement in man. On the environment, the steroid is either biodegraded or mineralized. It must be stated that escapee from tilapia hatchery into the natural water may, however, alter the dynamics of the environment due to unforeseen consequences.

Keywords: Sex-reversal, tilapia, man, environment

\section{Introduction}

T

ilapia has become the second most important finfish in aquaculture after carps with global production reaching 3.6 million tons in 2008 an increase from 2.5 million tons in 2007 (Megbowon, 2011). Because of their large size, rapid growth, and palatability, a number of tilapiine cichlids are at the focus of major aquaculture efforts. The main species cultured in ponds, cages and pens is the Nile tilapia (Oreochromis niloticus). The problem with this fish is their early maturation and ability to breed every month. These characteristics result in the overpopulation of stocked tilapia ponds and the stunting of growth because of the crowding of the fish (Fashina-Bombata and Megbowon, 2012). Another problem associated with a mixed sex of tilapia is the sizes of the fish at harvest, varying from small to large due to the faster growth of males. This makes it more difficult to establish uniformity of product. For producers wanting high yields of large-sized fish in 6 months, all male fry are preferred.

In 1960, only two countries in Africa had record of tilapia production. They were Egypt (2,100 MT) and Nigeria $(1,299 \mathrm{MT})$ (El-Sayed, 2006). It is however sad today, that tilapia production in Egypt exceeds 500,000MT while Nigeria produces about 50,000 MT (FDF, 2007). Presently, the local demand of tilapia in Nigeria is high but farmers lack the technical skill of producing large size of the fish under culture condition (Megbowon et al., 2009, Megbowon, 2011). The success of tilapia production in Egypt stemmed from the drive to ensure monosex male production through sex reversal using $17 \alpha$ methyl testosterone. Methyl testosterone treatment of tilapia fry is the most simple and reliable way to produce all male tilapia stocks, which consistently grow to a large/more uniform size than mixed sex tilapia.

All male culture of tilapia is preferred because of their faster growth than females (Megbowon, 2010). Production of all male tilapia can be accomplished by such techniques as separating the males and females manually, hybridization, chromosomal manipulation and hormonal sex reversal. The most efficient and least expensive method is sex reversal with the use of $17 \alpha$-methyl testosterone. There is however a global concern of the effect of this steroid on the fish flesh, consumers (man) and the environment (water bodies into which effluent is released) which forms the basis of this review. 


\section{Effects of methyl testosterone (MT) on fish, man and environment.}

In tilapia, sex reversal involves the treatment administration of male steroid to recently hatched fry so that the undifferentiated gonadal tissue of generic female develops testicular tissue, thus functioning reproductively as males (Megbowon et al., 2009).

\section{Houn} a problem created for the marketers of fish treated with hormone drugs. For instance marketing of treated fish is illegal in the EU countries and in India (White et al., 2006).

The hazard associated with the administration of MT in the culture of tilapia can be grouped into three-

i. Risk on tilapia consumers (man)

ii. Tilapia culturist

iii. Environment.

Risk of MT on tilapia consumer: Research findings have consistently shown that sex reversing tilapia with MT does not lead to accumulation of the hormone in the fish flesh after cessation of hormone treatment (Megbowon, 2011). Johnstone et al. (1983) revealed that whole fish body levels of MT were not detectable 100 hours after withdrawal of the hormone treated diet. Guerrero (2008,) further reported that hormone levels in tilapia falls to normal level five days after hormone feeding was stopped. Based on these and other scientific evidences, it clear that MT is rapidly removed from fish and will not persist after several months of eulture to market size. A conclusion can be drawn that the quantities of MT eaten by tilapia during sex inversion/reversal (fry treatment) is equivalent of $0.0-0.2 \mathrm{mg} \mathrm{MT} /$ fish represent less than $0.001 \%$ of the typical daily dosage of MT prescribed in human medicine $(20-40 \mathrm{mg}$ ) and that even this minute quantities declines to less than $0.00001 \%$ of the daily human dosage a week after eessation of hormone treatment. It has been reported that the testes of an adult man releases about $15 \mathrm{mg}$ of endogenous testosterone per day while about $10 \mathrm{mg}$ of androgen are excreted daily (Shore and Shemesh, 2003). These do not harm man.

The short treatment duration and rapid metabolism of MT help ensure that tilapia is free of MT before fish reach the consumer. Digested MT is rapidly metabolized and excreted. This rapid metabolism and excretion of MT by a fish treated early in its life history, combined with the extended period needed to produce a marketable size fish results in a safe consumer product.

However, as Nigeria is beginning to see the need to invest in tilapia culture and the drive for sex reversal is on the top gear to increase tilapia production, there may be need for caution. Although many workers have reported that the hormone is no more detectible in the muscle of the fish after two months of cessation of the hormone treatment, it is necessary to look beyond the muscle and examine some vital organs such as the liver and gills which have the propensity to accumulate substances. Furthermore, the product of biodegradation/mineralization of MT should be assayed for possible effect on the organs and metabolic parameters.

Effect of MT on Tilapia culturist: Tilapia culturists purchase commercially available androgen in powder or tablet form. This hormone is usually added to powdered tilapia feed which the culturist offers to tilapia fry many times per day during sex reversal using hormone treatment (FAO, 2006, Guerrero, 2008). The culturist or his workers may come into contact with the hormone in two ways: (1) when it is added to tilapia fry feed and (2) when MT treated feed is administered to tilapia fry in hatchery tanks. The hazard to fish farm worker ean be minimized by following the standard for handling such substances (OSHA, 2008, Syndel, 2008). Use of protective surgical gloves and face mask are strongly recommended to reduce the risk of inhalation of the hormone or entry through the skin contact.

Although the use of MT in commercial tilapia production is becoming more and more common globally, there has not been any report of adverse effect on farm workers. A more likely hazard that may arise from intentional ingestion of MT by staff is the well recognized anabolic (muscle building) and perceived libido-enhancing properties of the hormone.

Effect of MT on the environment: There is an increasing coneern about the implication of pharmaceutical active compounds such as hormones when effluent (wastewater) is discharged into the environment (Heberer, 2002). If all the projected tilapia production in 2010 of 3 million tons was produced through MT treated fish, the hormone used would not have been more than $100 \mathrm{~kg}$. In comparison, 33 tons of estrogen and 7.1 tons of androgen are excreted annually by farm animals in the European Union and 49 and 4.4 tons réspectively in USA (Lange et al., 2002). Wastewater discharge. from tilapia hatchery facilities represent only a tiny fraction of the total waste discharge into the environment but nonetheless, a precautionary approach should be taken. Lagoon and other natural waters are known to be highly effective in reducing concentration of pharmaceutical compounds via a combination of photolysis, plant uptake, microbial degradation and soil resorption (White et al., 2006). Steroid hormone in water are quickly absorbed into sediments or reduced to inorganic compound through mincralization (Shore and Semesh, 2003). For instance, bio-solids from municipal sewage treatment plant were found to rapidly mineralize added testosterone, equivalent to its more than $90 \%$ removal from the aqueous phase within 24 hours (Layton et al., 2000).

One major concern therefore to the environment is the tilapia escapee. Tilapia fry that escape from hatchery facilities where MT is administered will be morphologically male and consequently, they will have much lower potential to reproduce, 
including interbreeding with wild stock and this may alter the dynamics of the ecosystem. It is well established that reproductive potential of tilapia production is determined by the number of reproductively active females rather than the number of males. MT treatment eliminates the reproductive capacity of virtually all genetically female fish (Shore and Shemesh, 2003).

Furthermore, It has been shown that MT could accumulates in sediments of ponds, reaching 2 to $6 \mu \mathrm{g} / \mathrm{g}$ at 28 days after the onset of feeding with MT-impregnated food, and remained detectable in the soil between 2.8 and $2.9 \mu \mathrm{g} / \mathrm{g}$ after 84 days (eight weeks after ending treatment with MT-impregnated food), which demonstrated persistence of MT in soil for nearly three months after cessation of treatment (Contreras- Sánchez et al., 2000; Fitzpatrick et al., 2000). If this effluent is released into our natural waters, it may have a far-reaching effect of possibly sex reversing some natural population, thus altering the sex dynamics of the stock, producing more males than female which on the long run may lead to recruitment failure (Megbowon, 2011).

\section{Conclusion}

Production of male tilapia through the use of androgens is very effective. It does not require that a portion of the production be discarded as in manual selection, or that 2 separate stocks of fish be maintained as in hybridization. There are several seed production techniques adaptable to most scales of production. The relative ease and predictability of tilapia sex reversal has been a major factor in the rapid growth of the industry. Although a variety of hormones have been used for sex reversal, 17 $\alpha$-methyl testosterone is the most commonly used androgen. Dose rate and treatment durations vary depending on the environment and the experience of the producer.

It has been demonstrated that the hormone does not have any adverse effect on fish flesh after ecssation of treatment of tilapia fry. Ingestion of fish produced by sex reversal does not equally harm man. However, one is not too sure of the effect of the hormone or its bye products on vital organs (liver, kidney, pancreas and gills) metabolic profile and nucleic acids. On the environment, the steroid is either biodegraded or mineralized. It must be stated that escapee from tilapia hatchery into the natural water may alter the dynamics of the environment duc to unforesecn consequences

\section{REFERENCES}

FAO (2006). Aquaculture production statistics 1997-2006. Rome, Italy. 239pp.

Fashina-Bombata, H.A and Megbowon, I. (2012). Proximate composition and breeding description of an unidentified cichlid of Epe lagoon, Lagos, southwest Nigeria, commonly called "wesafu." Int'l Joumal of Nutrition \& Metaholism. 4(4): 57-63.

Guerrern, R,D.. III (2008). Tilapia sex reversal. www.agribusincssweek.com/tilpia-sex-reversal.

Herberer, T. (2002). Occurrence, fate and removal of pharmaceutical residues in the aquatic environment: $A$ review of recent research data. Tixicol, Leth, 131:5-17

Johnstone, R., Macintosh, D.J. and Wright, R.S. (1983). Elimination of orally administered $17 \alpha$ methyl testosterune by Oreochromis mossambicus (tilapia) and Salm gaidneri (Rainbow trout) juveniles. Aquaculture 35: 249257.

Lange, I.G., Daxenberger, A., Schiffer, B., Witters, H., lherrata, D., Meyer, H. H. D. (2002). Sex hormone originating from different livestock production systems: Fate and potential distupting activity in the Environment. Anal. Chm. Acta. 373: 27-37.

Layton, A.G, B.W., Gregory, J.R.Seward, T.W. Schultz and G.S. Sayler (2000). Minetalization of steroidal hormone by biosolids in wastewater treatment systems in Tennessec, USA. Environ. Sci. Technolo. 34: 3925-3931.

Mcgbowon, I., Fashina-Bombata, H.A., Mojekwu, T.O. and Okuade O.A. (2009). Genetic improvement of tilapia: challenges and prospects in Nigeria. Nigerian Journal of Fisheries. Vol.6 (1\&2), 21-30.

and Fashina-Bmbata, H.A. (2010). Tilapia: fish for the millennium. Fishnetwork, Vol. 3. Fisherics Society of Nigeria quarterly Publication (Supported by World Bank/NSME Nigeria). 36-40.

(2011). Tilapia production in Nigeria. Fishnetwork Vol. 4. $18-22$.

Shore, L.S. and Shemesh, M. (2003). Naturally produced steroid hormones and their release into the environment. Pure and Applied Chemistry 75: 1859-1871.

Syndel, (2008). www.syndel.com/msds/safehandling.html.

White, J.R., Belmont, M.A. and Metcalfe, C. D. (2006). Pharmaceutical compotunds in wastewaler: wetland treatment as a potential solution, 234-239.

OSHA (2008). www.osha.gov/dts/osta/otmvi'otmvi 2 .html. 